\title{
Isopropylphenidate: An Ester Homolog of Methylphenidate with Sustained and Selective Dopaminergic Activity and Reduced Drug Interaction Liability
}

\author{
John S. Markowitz, PharmD, ${ }^{1,2}$ Hao-Jie Zhu, $\mathrm{PhD}^{3}$ and Kennerly S. Patrick, $\mathrm{PhD}^{4}$
}

\begin{abstract}
Objective: The most widely utilized pharmacological treatment of attention-deficit/hyperactivity disorder (ADHD) is the psychostimulant methylphenidate (MPH). Most MPH formulations consist of the racemic mixture of $d$-threo-( $R, R)$-MPH and $l$-threo-( $S, S)$-MPH isomers. MPH is characterized by its low bioavailability and short half-life (2-3 hours). Additionally, significant inter-individual variability in MPH pharmacokinetics has been consistently documented. Accordingly, efforts have been directed at developing alternatives to MPH as therapeutic agents. A wide range of MPH analogues $(d l-\alpha-[2-$ piperidyl]-phenylacetic acid esters) have been synthesized with the dopamine transporter (DAT) and norepinephrine transporter (NET) as principle neuropharmacological targets. The present study investigated the metabolic profiles and pharmacological activity of the isopropyl ester derivative of MPH, $d l$-isopropylphenidate (IPH), both in vitro and in vivo.

Methods: The synthesis, monoaminergic transporter binding, cellular uptake profiles, and assessment of metabolic hydrolysis and transesterification in the presence of ethanol are described using MPH as a comparator. Additionally, an in vivo assessment of IPH stimulant effects (vs. saline) in rats was performed with locomotor activity as a pharmacodynamic outcome. Results: IPH displayed unique pharmacological characteristics including greater DAT than NET binding and cellular uptake activity, and greater resistance to hydrolysis and transesterification via carboxylesterase 1 relative to MPH. Further, sustained psychostimulant properties offer the prospect of an enhanced duration of action.

Conclusions: Our findings are consistent with IPH exhibiting attributes distinguishing it from MPH and warranting further study and development of IPH as a novel psychotherapeutic agent.
\end{abstract}

\section{Introduction}

A TTENTION-DEFICIT/HYPERACTIVITY DISORDER (ADHD) is a complex neurobehavioral disorder characterized by varying degrees of inattention, hyperactivity, and impulsivity (Biederman 2005). It is perhaps the single most common chronic health problems affecting school-age children, with an estimated worldwide prevalence of $8-12 \%$ (Faraone et al. 2003). The most widely utilized pharmacological treatment of ADHD is the psychostimulant methylphenidate $(\mathrm{MPH})$ consisting of the racemic (50:50) mixture of $d$-threo- $(R, R)-\mathrm{MPH}$ and $l$-threo- $(S, S)$-MPH isomers (Fig. 1). $\mathrm{MPH}$ is generally an effective and well-tolerated treatment that has been in clinical use for $>50$ years. MPH is widely available as an immediate-release (IR) tablet and displays a short plasma halflife of 2-3 hours as a result of rapid and stereoselective metabolism via de-esterification (Patrick et al. 2005a). This hydrolytic process is mediated by hepatic carboxylesterase 1 (CES1) in humans, yielding the major, albeit inactive, metabolite ritalinic acid (RA) that typically attains blood concentrations 30-60 times those of the parent compound (Patrick and Markowitz 1997). Use of IR formulations of MPH result in a relatively brief duration of action necessitating multiple daily doses to achieve symptom control throughout the day (Markowitz et al. 2003; Patrick et al. 2005a). However, administering MPH throughout the day poses problems in terms of convenience, compliance, security against diversion, and patient self-esteem, particularly for the school-age child (Greenhill et al. 2002). Accordingly, common clinical practice in the United States is to prescribe one of several modified-release (MR) pharmaceutical formulations (Markowitz et al. 2003) that vary in duration of action and release profile. Therefore, MR formulations of MPH have largely supplanted the use of IR formulations as the primary dosage forms employed in the treatment

\footnotetext{
${ }^{1}$ Department of Pharmacotherapy and Translational Research, University of Florida, Gainesville, Florida.

${ }^{2}$ Center for Pharmacogenomics, University of Florida, Gainesville, Florida.

${ }^{3}$ Department of Clinical, Social, and Administrative Sciences, University of Michigan College of Pharmacy, Ann Arbor, Michigan.

${ }^{4}$ Department of Drug Discovery and Biomedical Sciences, Medical University of South Carolina, Charleston, South Carolina.
} 


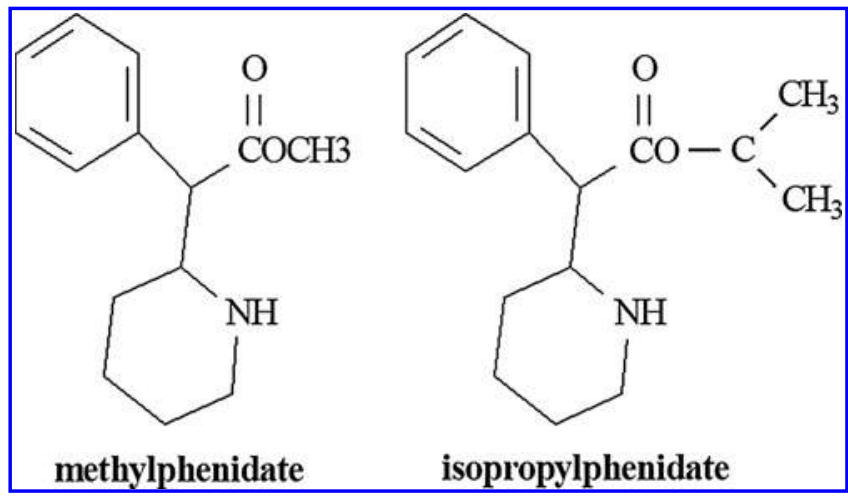

FIG. 1. Chemical structures of methylphenidate (MPH) and isopropylphenidate (IPH).

of ADHD. Although the half-life parameter of the MPH remains unchanged upon release from such formulations, this approach can provide the often necessary all-day symptom "coverage" with a single daily dose. Despite these advances in biopharmaceutical delivery of MPH, in many instances these formulations are not successful for patients because of inter-individual physiological differences in drug absorption as such MR formulations pass the various regions of the gut (McConnell et al. 2008), or are prohibitively expensive. Significant inter-individual variability in response to MPH pharmacotherapy has been consistently documented in clinical research literature. For example, up to $30 \%$ of patients receiving MPH do not derive adequate therapeutic effects, or experience treatment-limiting side effects (Patrick et al. 2005a). The variation in therapeutic outcomes have been partially attributed to significant variation of CES1 activity and expression associated with CES1 genetic variants (Zhu et al. 2008) and environmental factors (i.e., CES1 inhibitors and/or inducers). Therefore, MPH congeners that are similar in terms of pharmacologic activity, yet resistant to CES1 hydrolysis, have emerged as an area of active drug development. Ideally, such compounds will extend the duration of action and provide more predictable clinical outcomes as a result of reduced inter-individual variability of CES1-medicated drug metabolism.

In the present study, the MPH homolog $d l$-isopropylphenidate (IPH) (Fig. 1) was synthesized and assessed for its pharmacological activity and metabolic profiles. Monoamine transporter binding and cellular uptake experiments were performed on racemic IPH, as well as on the comparators MPH and the ethyl ester congener previously characterized by our group, $d$-ethylphenidate (EPH) (Patrick et al. 2005b). Additionally, an assessment of metabolic hydrolysis of the three compounds by CES1 was performed utilizing CES1 transfected cell lines, human liver microsomes (HLM), and human intestinal microsomes (HIM). Furthermore, the potential for a CES1-mediated transesterification reaction between IPH and ethanol to ethylphenidate was evaluated and compared with that of MPH in vitro, in view of the metabolic drug interaction associated with the MPH-ethanol combination (Patrick et al. 2007). Finally, an in vivo assessment of IPH stimulant effects (vs. saline) on locomotor activity was performed utilizing experimental methodology previously employed to establish the in vivo locomotor activity of the separate $d$ - and $l$-isomers of MPH in rats (Patrick et al. 1987). The overarching goal of this series of experiments was to explore the pharmacological characteristics of IPH as a potential new therapeutic entity.

\section{Materials and Methods}

\section{Chemical compounds and animals}

MPH was purchased from Sigma-Aldrich (St. Louis, MO). EPH synthesis was conducted in our laboratory as previously described (Patrick et al. 2005b). IPH was synthesized in our laboratory using the following method: Briefly, ( \pm )-ritalinic acid $(2 \mathrm{mM})$ was dissolved in isopropyl alcohol saturated with $\mathrm{HCl}$ gas $(75 \mathrm{~mL})$ and refluxed for 24 hours under nitrogen. The solution was evaporated to dryness under reduced pressure and purged with nitrogen, and the white residue was then dissolved in a minimum volume of warm isopropyl alcohol. Diethyl ether was then added to turbidity and the flask was stored for 24 hours at $2^{\circ} \mathrm{C}$. The resulting white crystalline product was filtered, washed with diethyl ether, and dried under vacuum. The purity of the synthetic material was confirmed by gas chromatographymass spectrometry. All compounds studied were assessed as their $\mathrm{HCl}$ salts. The remaining reagents and solvents were of the highest grade commercially available. Animals used in these experiments were male Sprague-Dawley rats weighing 200-300 g and obtained from Charles River Laboratories (Wilmington, MA).

\section{Transporter binding and cellular uptake}

All described monoamine transporter binding studies as well as cellular uptake assays were performed in duplicate by CEREP (Celle l'Evescault, France) and are described subsequently. Further details of all assays performed may be accessed at the CEREP web site (www.cerep.com). Cellular-based assays were conducted to provide complementary in vitro functional measures to complement the standard transporter assays as well as to provide direct comparisons of the homologs IPH with MPH and EPH with regard to dopamine (DA), norepinephrine (NE), and 5-HT uptake in synaptosomal preparations. The concentration of MPH, EPH, and IPH utilized in these studies was $10 \mu \mathrm{M}$.

\section{Monoamine transporter radioligand binding assays}

Dopamine transporter radioligand binding assay. Evaluation of the affinity of all three compounds for the human DA transporter (DAT) in transfected Chinese hamster ovary (CHO) cells was determined in a validated radioligand binding assay. Cell membrane homogenates were incubated for 120 minutes at $4^{\circ} \mathrm{C}$ with $0.5 \mathrm{nM}\left[{ }^{3} \mathrm{H}\right]$ GBR 12935 in the absence or presence of each test compound in a standard buffer solution. Nonspecific binding was determined in the presence of $10 \mu \mathrm{M} \mathrm{N}$-(1-[Benzo[b]thien-2-ylcyclohexyl]) piperidine (BTCP). Following incubation, the samples were filtered under vacuum through glass filters and rinsed several times with ice-cold $50 \mathrm{mM}$ Tris- $\mathrm{HCl}$ using a 96 sample cell harvester. The filters were then dried and measured for radioactivity with a scintillation counter (TopCount, Packard) using a liquid scintillation cocktail (Microscint 0, Packard). The results of DAT binding experiments as well as the other transporter assays described in the following sections were expressed as a percent inhibition of the control radioligand specific binding. The standard reference compound was BTCP, which was tested in each experiment at several concentrations in order to obtain a competition curve from which its inhibitory concentration (IC) 50 was calculated.

Norepinephrine transporter radioligand binding assay. Evaluation of the affinity of compounds for the human NE transporter (NET) in transfected $\mathrm{CHO}$ cells was determined in a radioligand assay analogous to the procedures described for the DAT assay. Cell membrane homogenates were incubated for 90 minutes at 
$4^{\circ} \mathrm{C}$ with $1 \mathrm{nM}\left[{ }^{3} \mathrm{H}\right]$ nisoxetine in the absence or presence of each test compound in a standard buffer solution. Nonspecific binding was determined in the presence of $1 \mu \mathrm{M}$ of desipramine. Following incubation, the samples were filtered rapidly under vacuum and rinsed several times with a buffer solution. The filters were then dried, and radioactivity counts were obtained. The results are expressed as percent inhibition of the control radioligand specific binding. The standard reference compound was the tricyclic antidepressant (TCA) protriptyline, which was tested in each experiment at several concentrations to generate a competition curve from which its $\mathrm{IC}_{50}$ was calculated.

Serotonin transporter radioligand binding assay. An evaluation of the respective compounds was also conducted regarding their affinity for the human serotonin 5-HT transporter (SERT) in transfected $\mathrm{CHO}$ cells. Briefly, cell membrane homogenates were incubated for 90 minutes at $4^{\circ} \mathrm{C}$ with $2 \mathrm{nM}$ of $\left[{ }^{3} \mathrm{H}\right]$ imipramine in the absence and presence of each of the assessed compounds. Nonspecific binding was determined in the presence of $10 \mu \mathrm{M}$ of imipramine. After incubation, the samples were filtered rapidly under vacuum and rinsed several times with buffer solution. The filters were then dried and measured for radioactivity via scintillation counter. The standard reference compound was the TCA imipramine, which was tested in each experiment at several concentrations to generate a competition curve from which its $\mathrm{IC}_{50}$ was calculated.

\section{Cellular-based monoamine uptake assays}

Norepinephrine uptake. The evaluation of the effects of each compound of interest (MPH, EPH, IPH) on NE uptake utilized synaptosomes prepared from the rat hypothalamus. These synaptosomes $(100 \mu \mathrm{g})$ were incubated for 20 minutes at $37^{\circ} \mathrm{C}$ with $0.1 \mu \mathrm{Ci}$ $\left[{ }^{3} \mathrm{H}\right]$ norepinephrine in the absence (i.e. control) or presence of the test compound or the reference compound in a standard buffer solution. Basal control activity was determined by incubating the same mixture for 20 minutes at $0^{\circ} \mathrm{C}$ in the presence of $10 \mu \mathrm{M}$ protriptyline to block the uptake. Following incubation, the samples were filtered, counted using a scintillation instrument, and the results expressed as a percent inhibition of the control uptake of $\left[{ }^{3} \mathrm{H}\right]$ norepinephrine. The standard inhibitory reference compound was the TCA protriptyline, which was tested in each experiment at several concentrations to obtain an inhibition curve from which its $\mathrm{IC}_{50}$ value was calculated.

DA uptake. The evaluation of the effects of the three compounds on DA uptake again utilized synaptosomes, but this time prepared from the rat striatum. The synaptosomes were incubated for 15 minutes at $37^{\circ} \mathrm{C}$ with $0.1 \mu \mathrm{Ci}\left[{ }^{3} \mathrm{H}\right]$ dopamine in the absence and presence of the test compound or the reference compound in a buffer standard buffer solution. Basal control activity was determined by incubating the same mixture for 15 minutes at $4^{\circ} \mathrm{C}$ in the presence of $1 \mu \mathrm{M}$ GBR12909 to block the uptake. After incubation, the samples were filtered, counted, and the results expressed as a percent inhibition of the control uptake of $\left[{ }^{3} \mathrm{H}\right]$ dopamine by scintillation count. The standard inhibitory reference compound was GBR12909, which was tested in each experiment at several concentrations to obtain an inhibition curve from which its $\mathrm{IC}_{50}$ value was calculated.

Serotonin uptake. The assessment of the effects of the three compounds on 5-HT uptake utilized measures of $\left[{ }^{3} \mathrm{H}\right] 5$-HT incorporation into synaptosomes prepared from the rat brain. The synaptosomes were incubated for 15 minutes at $37^{\circ} \mathrm{C}$ with $\left[{ }^{3} \mathrm{H}\right] 5-\mathrm{HT}$
$(0.2 \mu \mathrm{Ci} / \mathrm{mL})$ in the absence and presence of each of the three assessed compounds or the reference compounds. Following incubation, the samples were filtered, counted using a scintillation instrument, and the results expressed as a percent inhibition of the control uptake of $\left[{ }^{3} \mathrm{H}\right] 5-\mathrm{HT}$. The standard inhibitory reference compound was the TCA imipramine, which was tested in each experiment at several concentrations to obtain an inhibition curve from which its $\mathrm{IC}_{50}$ value was calculated.

\section{Determination of relative hydrolytic rates of $M P H, E P H$, and IPH}

The assessment of relative rates of hydrolysis of the three ester homologs utilized CES1 transfected cell lines, HLM, and HIM. The s9 fractions from the HEK293 cells transfected with human CES1A1 gene were prepared utilizing a method developed in our laboratory and described previously (Zhu et al. 2008). Hydrolysis of IPH, EPH, and MPH was assessed by measuring the formation of the hydrolytic metabolite RA following incubation with cell s9 fractions, HLM, and HIM. Briefly, $50 \mu \mathrm{L}$ of freshly prepared substrates (racemc IPH, EPH, and MPH) was mixed with $50 \mu \mathrm{L}$ of enzymes in $1.5 \mathrm{~mL}$ Eppendorf tubes. Both substrates and enzymes were diluted in Dulbecco's Phosphate-Buffered Saline (DPBS, $20 \mathrm{mM}$ HEPES, $\mathrm{pH}$ 7.4). The final substrate concentration was $1 \mathrm{mM}$, and the final enzyme concentrations were $0.5 \mathrm{mg} / \mathrm{mL}$, $0.2 \mathrm{mg} / \mathrm{mL}$, and $0.2 \mathrm{mg} / \mathrm{mL}$ for the s9 fractions, HLM, and HIM, respectively. Following incubation at $37^{\circ} \mathrm{C}$ for 60 minutes, the reaction was terminated by adding $500 \mu \mathrm{L}$ of methanol. The samples were then centrifuged at $16,000 \mathrm{~g}$ at $4^{\circ} \mathrm{C}$ for 5 minutes to remove precipitated protein. The concentrations of the common hydrolytic metabolite RA of all three compounds were determined utilizing a validated high-performance liquid chromatography (HPLC) method we previously described (Zhu et al. 2008).

\section{CES1 catalyzed transesterification IPH and $\mathrm{MPH}$ in the presence of ethanol}

The working solutions of the s9 fractions of CES1 transfected cells and the substrates (MPH, IPH, and ethanol) were prepared in DPBS containing $20 \mathrm{mM}$ HEPES ( $\mathrm{pH} 7.4)$. The reaction was initiated by mixing $200 \mu \mathrm{L}$ of the s 9 fractions, $100 \mu \mathrm{L}$ of MPH or IPH, and $100 \mu \mathrm{L}$ of ethanol. The final concentrations of the $\mathrm{s} 9$ fraction protein, MPH, IPH, and ethanol were $2 \mathrm{mg} / \mathrm{mL}, 1 \mathrm{mM}, 1 \mathrm{mM}$, and $10 \mathrm{mM}$, respectively. After incubation at $37^{\circ} \mathrm{C}$ for $1 \mathrm{~h}$, the reaction was terminated by adding a fourfold volume of ice-cold methanol. The samples were then briefly vortexed and centrifuged at $16,000 \mathrm{~g}$ for 5 minutes at $4{ }^{\circ} \mathrm{C}$, and the resulting supernatant was subjected to HPLC analysis to assess the concentrations of the transesterification product EPH from both MPH and IPH, and common metabolite RA. The s9 fractions prepared from the vector transfected cells were included as a negative control for any nonenzymatic hydrolysis that might occur. The CES1-mediated metabolism of MPH and IPH (hydrolysis and transesterification) were estimated by substracting the amounts of RA and EPH produced in the vector s9 samples from that observed in the CES1 s9 fraction samples.

\section{Locomotor activity measurement in rats}

Locomotor-inducing activity of IPH was measured according to methods previously described for the differential pharmacology of MPH enantiomers (Patrick et al. 1987). The present study compared only the IPH homolog versus saline dosing since the other compounds have been extensively investigated in vivo previously 
Table 1. Transporter Binding Affinity Study of MPH, EPH, and IPH for the Major Monoamine Transporters

\begin{tabular}{|c|c|c|c|c|c|}
\hline \multirow[b]{2}{*}{ Monoamine transporter } & \multirow[b]{2}{*}{ Ligand } & \multirow{2}{*}{$\begin{array}{l}\text { Reference } \\
\text { compound }\end{array}$} & \multicolumn{3}{|c|}{$\begin{array}{l}\text { Compound assessed }(10 \mu \mathrm{M}) \\
\text { (\% inhibition of control specific binding) }\end{array}$} \\
\hline & & & $I P H$ & $\mathrm{MPH}$ & $E P H$ \\
\hline Dopamine (DAT) $(h)$ & {$\left[{ }^{3} \mathrm{H}\right] \mathrm{GBR}-12935$} & BTCP & 97 & 101 & 97 \\
\hline Norepinephrine (NET) $(h)$ & {$\left[{ }^{3} \mathrm{H}\right]$ nisoxetine } & Protriptyline & 27 & 88 & 45 \\
\hline Serotonin (SERT) $(h)$ & {$\left[{ }^{3} \mathrm{H}\right]$ imipramine } & Imipramine & 20 & 6 & 29 \\
\hline
\end{tabular}

BTCP, N-[1-(Benzo[b]thien-2-yl-cyclohexyl)]piperidine; [3H]GBR-1293, 1-[2-(Diphenylmethoxy)ethyl]-4-(3-phenylpropyl)piperazine; MPH, racemic (dl) methylphenidate; $\mathrm{EPH}$, racemic ethylphenidate; IPH, racemic isopropylphenidate.

(Williard et al. 2007). In brief, following an initial 60 minute habituation period to the activity chamber, racemic IPH (or saline) was administered intraperitonealy (i.p.) to each animal $(n=5$ per active drug group; $n=3$ per saline group) at a dose of $10 \mathrm{mg} / \mathrm{kg}$ to correspond with $10 \mathrm{mg}$ of racemic MPH (delivering $5 \mathrm{mg}$ of $d$ MPH), which was previously determined to produce near maximal behavioral responses (Patrick et al. 1987). Locomotor activity was recorded within doughnut-shaped cages with six photocell sensors equally spaced around a $9 \mathrm{~cm}$ runway. Activity counts as assessed by light beam interruptions were recorded for each animal in increments of 10 minutes over a 2 hour period. Differences in the cumulative motor activity accounts were assessed by the unpaired, two tailed Student $t$ test. The level of significance was set at $p<0.05$.

\section{Results}

\section{Monoamine transporter binding and cellular} uptake studies

The binding of racemic IPH, MPH, and EPH to the prominent cellular monoamine transporters DAT, NET, and SERT revealed that, as a group, binding affinities were greatest for DAT. All compounds produced significant effects at the DAT, with insignificant differences noted among the three compounds (Table 1). With regard to NET, as anticipated, MPH exhibited substantial binding as measured by inhibition of specific control binding, whereas EPH exhibited approximately half the binding affinity of $\mathrm{MPH}$, and IPH was the lowest at a value approximately one third that of MPH at the tested concentration $(10 \mu \mathrm{M})$. In the case of SERT, none of the compounds assessed either approached or exceeded $50 \%$ inhibition of control specific binding.

The results of monoamine cellular (i.e., functional) assays are presented in Table 2. With regard to DA, all three compounds exhibited significant effects on the uptake of this monoamine, with little difference observed among the agents. Norepinephrine uptake studies indicated that MPH and EPH exerted the most effects, whereas IPH was significantly lower at the concentration of $10 \mu \mathrm{M}$.
Finally, 5-HT uptake was not affected to any significant degree by any of the three assessed compounds.

\section{Hydrolysis of IPH, EPH, and $M P H$}

CES1 is highly expressed in the human liver, and is purported to account for the majority of hydrolytic activity within the organ, whereas CES2 is the predominant hydrolase in the human intestine (Kagan and Hoffman 2008). In the present study, the susceptibility of $\mathrm{MPH}, \mathrm{EPH}$, and IPH to CES1-mediated hydrolysis was investigated in parallel by incubation of each substrate with the cell s9 fractions of CES1 transfected cells as well as HLM and HIM preparations. The results indicated that the catalytic efficiency of CES1-mediated hydrolysis upon MPH is approximately 10-fold higher than for IPH (Fig. 2). When HLM preparations were utilized for incubations, EPH appeared to be the most vulnerable substrate relative to MPH and IPH, whereas IPH was the most resistant substrate to HLM (Fig. 2). Data from the HIM incubation studies where CES2 (but not CES1) was extensively expressed indicated that EPH was an efficient substrate of CES2. In contrast, catalytic activity of HIM was extremely low with regard to both MPH and IPH hydrolysis (Fig. 2).

In summary, the present data demonstrate that IPH is a poor substrate of CES1. Nevertheless, albeit at a markedly lower rate, IPH was predominantly metabolized (i.e., hydrolyzed) by CES1, with little to no contribution from CES2, an observation similar to that which is known to be the case with MPH and established structure-activity relations for hCES1 and its ester substrates (Ross and Crow 2007).

\section{Transesterification potential of IPH versus $\mathrm{MPH}$}

MPH was efficiently converted to EPH via CES1 catalyzed transesterification in the presence of ethanol, with a velocity of $423.3 \pm 44.4 \mathrm{pmole} / \mathrm{min} / \mathrm{mg}$ protein. In contrast, IPH displayed significant resistance to CES1-mediated transesterification, exhibiting a velocity of $47.5 \pm 3.3 \mathrm{pmole} / \mathrm{min} / \mathrm{mg}$ protein (Fig. 3). Furthermore, no EPH formation was observed following the incubation of MPH and IPH with ethanol when CES1 was not

Table 2. Cellular Uptake Studies of MPH, EPH, and IPH

\begin{tabular}{|c|c|c|c|c|c|}
\hline \multirow{2}{*}{$\begin{array}{l}\text { Cellular uptake } \\
\text { assay substrate }\end{array}$} & & \multirow[b]{2}{*}{ Measured parameter } & \multicolumn{3}{|c|}{$\begin{array}{c}\text { Compound assessed }(10 \mu M) \\
(\% \text { inhibition of control uptake) }\end{array}$} \\
\hline & & & $I P H$ & $M P H$ & $E P H$ \\
\hline Dopamine uptake & {$\left[{ }^{3} \mathrm{H}\right] \mathrm{DA}$} & {$\left[{ }^{3} \mathrm{H}\right] \mathrm{DA}$ incorporation into synaptosomes } & 96 & 90 & 90 \\
\hline Norepinephrine uptake & {$\left[{ }^{3} \mathrm{H}\right] \mathrm{NE}$} & {$\left[{ }^{3} \mathrm{H}\right] \mathrm{NE}$ incorporation into synaptosomes } & 62 & 117 & 116 \\
\hline Serotonin uptake & {$\left[{ }^{3} \mathrm{H}\right] 5-\mathrm{HT}$} & {$\left[{ }^{3} \mathrm{H}\right] 5$-HT incorporation into synaptosomes } & 28 & 35 & 48 \\
\hline
\end{tabular}

MPH, racemic ( $d l$ ) methylphenidate; EPH, racemic ethylphenidate; IPH, racemic isopropylphenidate. 


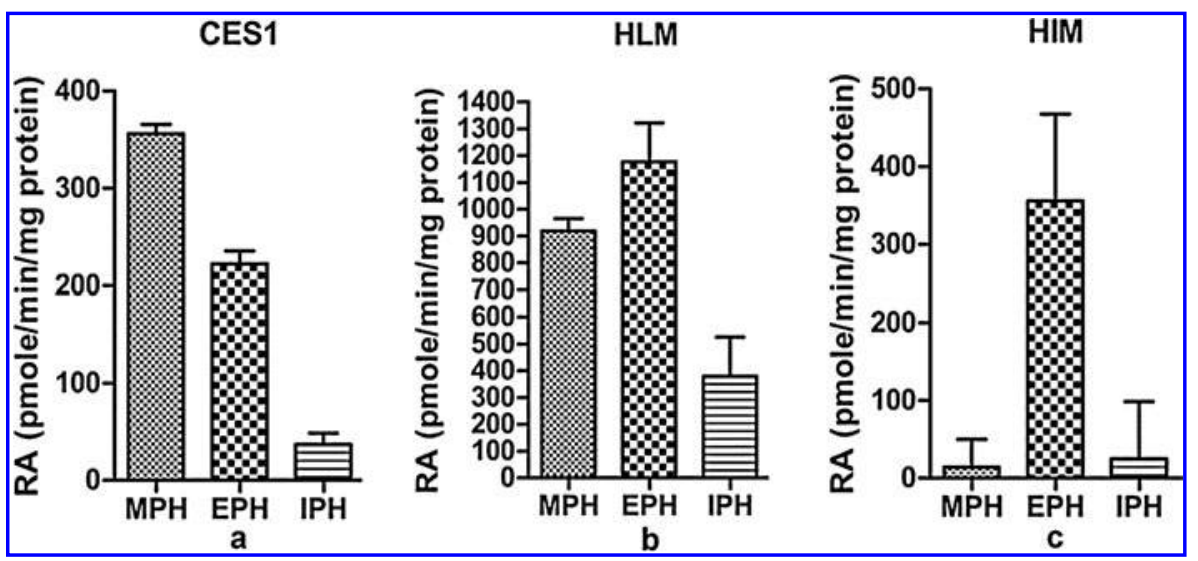

FIG. 2. Hydrolysis of methylphenidate (MPH), ethylphenidate (EPH), and isopropylphenidate (IPH) by carboxylesterase 1 (CES1) cell s9 fractions (a), human liver microsomes (HLM) (b), and human intestinal microsomes (HIM) (c). The metabolite ritalinic acid (RA) formed from the hydrolysis of MPH, EPH, and IPH was determined by a validated high-performance liquid chromatography (HPLC) assay following incubation with the s9 fractions, HLM, and HIM at $37^{\circ} \mathrm{C}$ for 60 minutes. Data are presented as mean \pm SD of three to six independent experiments.

present. In addition to yielding the novel transesterification metabolite EPH (Markowitz et al. 1999; Patrick et al., 2007), ethanol also significantly decreased the formation of the hydrolytic product RA following incubation with MPH.

\section{Rat locomotor activity}

The time course of locomotor response over a 120 minute period following i.p. administration of IPH $(10 \mathrm{mg} / \mathrm{kg})$ versus saline is shown in Figure 4a. As expected, IPH produced robust effects on locomotor activity in rats when compared with saline injections at 10 minute intervals recorded post-dosing, with a mean of nearly 1200 counts recorded during the initial 10 minute measurement. Also shown in Figure $4 \mathrm{~b}$ are the cumulative locomotor activity

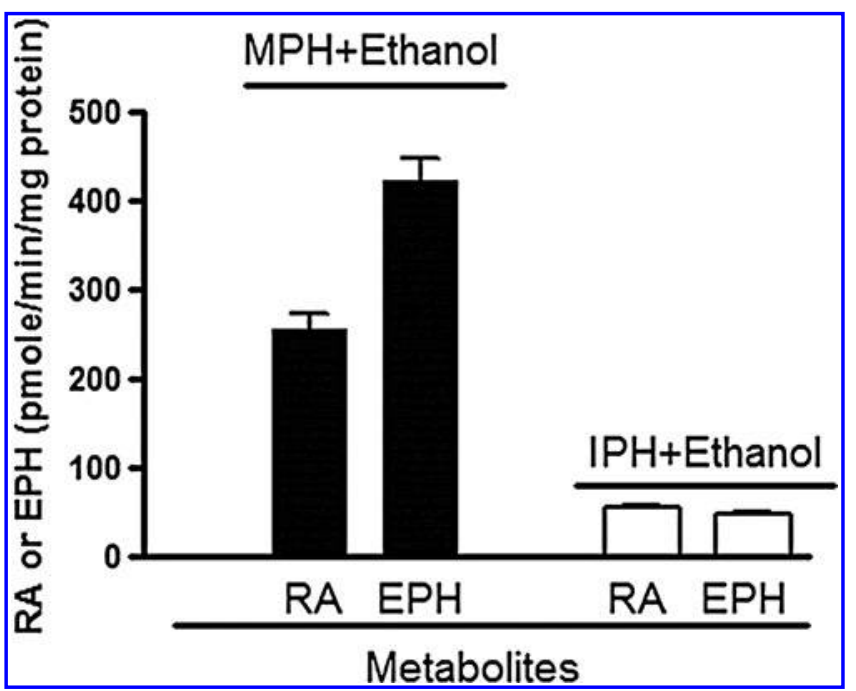

FIG. 3. Carboxylesterase 1 (CES1) catalyzed hydrolysis and transesterification of methylphenidate (MPH) and isopropylphenidate (IPH) in the presence of ethanol. The hydrolytic metabolite ritalinic acid (RA) and the transesterification product ethylphenidate (EPH) were determined after the incubation of $\mathrm{MPH}$ and IPH with CES1 cell s9 fractions in the presence of ethanol at $37^{\circ} \mathrm{C}$ for 60 minutes. Data are means from three independent experiments with error bars representing SD. counts over the entire 120 minute study period. Racemic IPH administration significantly elevated the cumulative locomotor activity counts in comparison with saline-injected rats $(p<0.01)$.

\section{Discussion}

The present study provides a systematic assessment of in vitro and in vivo pharmacological activity, including the catalytic hydrolysis and ethanolysis rates of CES1-mediated actions using the MPH ester homolog IPH. Evaluation of the binding affinities of IPH, MPH, and EPH for DAT, NET, and SERT revealed substantial differences among these ester congeners. Overall, the transporter binding data generated for the prototype "phenidate" compound, MPH, was consistent with the majority of published in vitro reports conducted using similar methodologies (Markowitz and Patrick 2008). All tested compounds showed similar and significant binding affinities for DAT with little interaction with SERT (Table 1). With regard to NET, IPH exhibited substantially less binding affinity than MPH which was the highest of the three compounds, and significantly less than EPH which exhibited modest binding affinity at NET when these compounds were tested at a single concentration of $10 \mu \mathrm{M}$. The results of the complementary cellular functional studies indicated that all tested agents produce a high degree of DA uptake inhibition and few differences in these action were noted among the three (Table 2). Uptake of NE was found to be significantly lower for IPH relative to both MPH and EPH at the concentration of $10 \mu \mathrm{M}$. Finally, 5-HT uptake was not significantly influenced by any of the agents, although EPH appeared to exert the greatest effect of the three compounds under the present experimental conditions.

Taken together, the preliminary pharmacological screening of monoamine transporter binding and cellular uptake studies suggest that IPH is primarily a dopaminergic compound with significantly less noradrenergic activity than MPH or EPH at the concentration of $10 \mu \mathrm{M}$. Although there is substantial evidence of a noradrenergic component in some cases of ADHD pathophysiology as well as MPH pharmacotherapy, the prevailing view is that often clinically significant cardiovascular side effects (increased heart rate and blood pressures) associated with MPH, as well as amphetamines, are primarily mediated by the noradrenergic, sympathomimetic component of these psychostimulant actions. Accordingly, an agent that appears to have less noradrenergic activity, such as IPH, could 


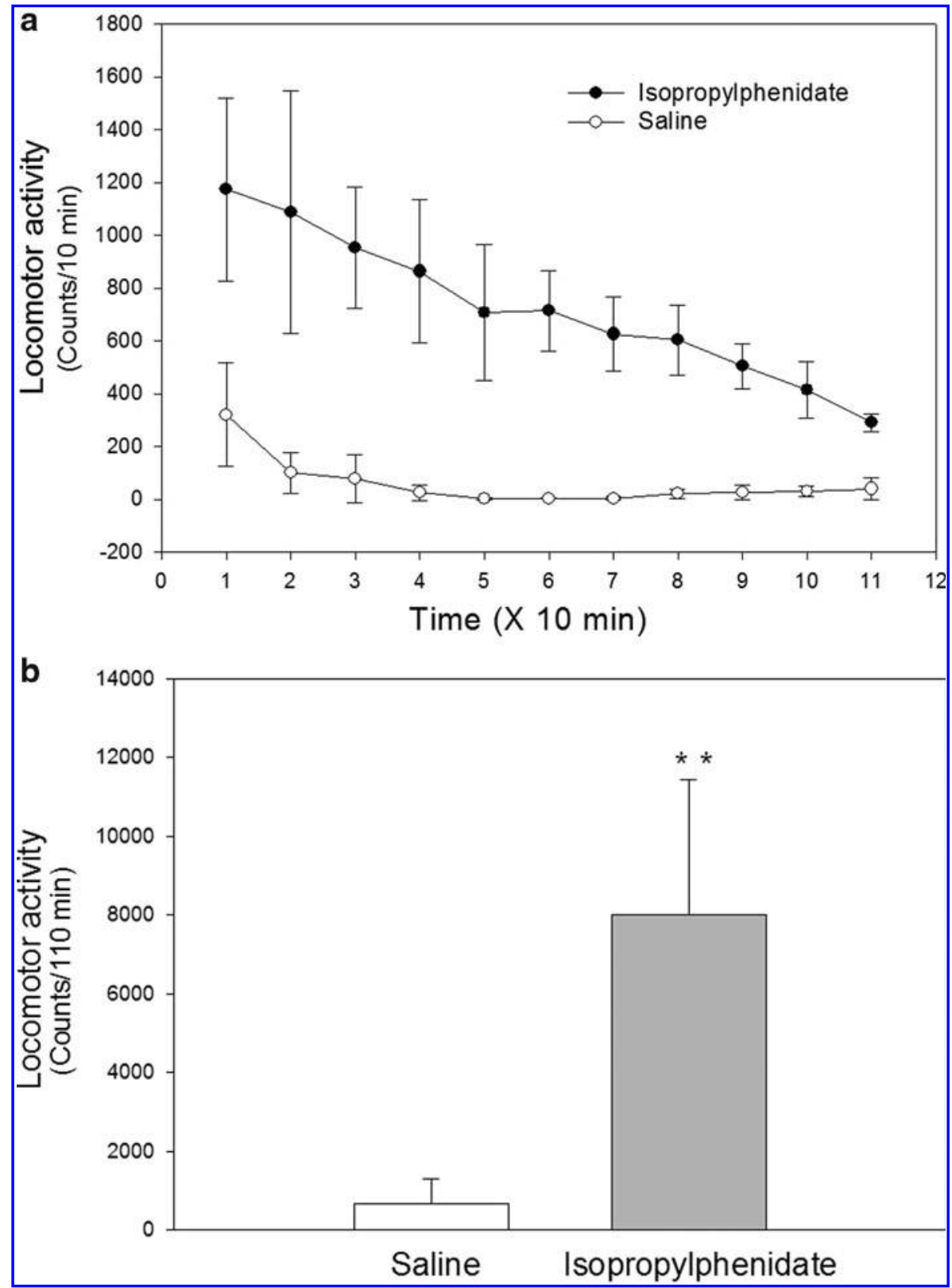

FIG. 4. Locomotor activity at 10 minute intervals (a) and during the entire study period (b) in rats intraperitoneally injected with isopropylphenidate (IPH) $(10 \mathrm{mg} / \mathrm{kg})$ or saline. ${ }^{* *} p<0.01$.

potentially provide an improved safety profile relative to existing drugs (e.g., MPH) presently employed in ADHD treatment.

The results of enzymatic hydrolysis experiments conducted using HLM, HIM, and transfected cells overexpressing CES1 also produced substantially different results for the IPH compound relative to $\mathrm{MPH}$ and $\mathrm{EPH}$. The data generated in the present study demonstrate that IPH is a relatively poor substrate of CES1, which is in contrast to what is observed for MPH and EPH (Figure 2). IPH was predominantly metabolized (i.e., hydrolyzed) by CES1 to RA, an inactive metabolite common to all three compounds, at a significantly lower rate relative to MPH and EPH. There was little to no contribution to IPH metabolism by CES2.

The pharmacologically active metabolite $d$-EPH can be formed in subjects co-ingesting MPH and ethanol, but it is not formed to the extent that the inactive $l$-EPH is formed (Patrick et al. 2005b, 2007). Co-abuse of MPH and alcohol has been well documented, and the recognized interaction between MPH and ethanol has become an area of concern in view of the associated elevation of $d$-MPH by ethanol (Patrick et al., 2007). Our in vitro incubation studies revealed that IPH displays great resistance to CES1-mediated transesterification, indicating that IPH has significantly reduced interaction potential when co-ingested with ethanol relative to MPH (Fig. 3).

The mechanism by which IPH is less efficiently hydrolyzed or transesterified relative to MPH can be speculated as based on the bulkier isopropyl substituent providing sufficient steric hindrance in accessing the active site of CES1 relative to MPH. This is consistent with current theory on structural requirements for CES1 substrates, which generally describes molecules esterified by a small alcohol group and also containing a large acyl group (e.g., MPH) (Imai 2006). It is noted, conversely, CES2 tends to show greater catalytic activity toward structures with larger alcohol groups and smaller acyl groups (Imai 2006). 
In the present investigation, i.p. administration of racemic IPH to rats produced potent locomotor activity effects, as consistent with the pharmacology of MPH and other DAT-active psychostimulants that have proven useful in the management of ADHD. These data add to existing in vitro data suggesting IPH to be a significantly active central nervous system (CNS) compound at $\mathrm{mg} / \mathrm{kg}$ doses similar to those used in assessments of MPH in eliciting classic stimulantinduced behavioral responses in rodents (Patrick et al. 1987). Furthermore, when compared with the earlier study by Patrick and associates (1987) characterizing the pharmacology of MPH isomers, racemic IPH dosed at $10 \mathrm{mg} / \mathrm{kg}$ appeared to produce more potent and sustained locomotor responses than those of $d$-MPH dosed at $5 \mathrm{mg} / \mathrm{kg}$.

\section{Conclusions}

The present report provides in vitro evidence that IPH displays pharmacological characteristics of a CNS stimulant with a high affinity for DAT, as well as potent effects on cellular uptake of DA. However, unlike MPH, IPH has only minor effects on NE, which could theoretically provide a more desirable safety/toxicity profile. Additionally, a substantially slower rate of enzymatic hydrolysis and transesterification via CES1 was noted relative to MPH, suggesting less potential of drug interaction with ethanol, a longer duration of action and an extended dosage interval could be utilized, which is viewed as necessary in the current treatment of ADHD with MPH. Finally, in vivo studies demonstrated potent stimulating effects on locomotor activity in IPH dosed rats similar to that typically observed following MPH or amphetamine dosing. Forthcoming dose-response and time course in vivo activity assessments incorporating direct comparisons with $\mathrm{MPH}$ and expanded in vitro investigation of monoamine activities (see Patrick et al. 2005b) will further advance the present preclinical investigations, setting the stage for potential new drug development.

\section{Clinical Significance}

MPH exhibits a relatively short half-life as a result of rapid hydrolytic metabolism catalyzed by hepatic CES1, which necessitates multiple daily doses, or the use of expensive MR formulations to achieve symptom control throughout the day. Additionally, significant inter-individual variability in response to MPH therapy has been consistently documented, which is, at least in part, because of varied CES1 expression and activity caused by genetic polymorphisms and environmental factors. The present study revealed that IPH is more resistant to CES1-catalyzed hydrolysis and transesterification reactions than MPH, indicating that IPH may offer a longer duration of action and less potential for drug-drug interactions via CES1, and that it will be the subject of addition preclinical studies.

\section{Acknowledgment}

The authors thank Dr. George R. Breese, University of North Carolina-Chapel Hill, in whose laboratory the locomotor activity study was conducted.

\section{Disclosures}

Dr. Patrick has been a consultant and expert witness with Johnson \& Johnson, and a consultant for Celgene, Janssen-Ortho, Novartis, Noven, and UCB. Drs. Markowitz and Zhu have no conflict of interests to declare.

\section{References}

Biederman J: Attention-deficit/hyperactivity disorder: A selective overview. Biol Psychiatry 57:1215-1220, 2005.

Faraone SV, Sergeant J, Gillberg C, Biederman J: The worldwide prevalence of ADHD: Is it an American condition? World Psychiatry 2:104-113, 2003.

Greenhill LL, Pliszka S, Dulcan MK: Practice parameters for the use of stimulant medications in the treatment of children, adolescents, and adults. J Am Acad Child Adolesc Psychiatry 41 (Suppl):26S-49S, 2002.

Imai T: Human carboxylesterase isozymes: catalytic properties and rational drug design. Drug Metab Pharmacokinet 21:173-185, 2006.

Kagan L, Hoffman A: Systems for region selective drug delivery in the gastrointestinal tract: biopharmaceutical considerations. Expert Opin Drug Deliv 5:681-692, 2008.

Markowitz JS, Logan BK, Diamond F, Patrick KS: Detection of the novel metabolite ethylphenidate after methylphenidate overdose with alcohol coingestion. J Clin Psychopharmacol 19:362-366, 1999.

Markowitz JS, Patrick KS: Differential pharmacokinetics and pharmacodynamics of methylphenidate enantiomers: Does chirality matter? J Clin Psychopharmacol 28:S54-61, 2008.

Markowitz JS, Straughn AB, Patrick KS: Advances in the pharmacotherapy of attention-deficit-hyperactivity disorder: Focus on methylphenidate formulations. Pharmacotherapy 23:1281-1299, 2003.

McConnell EL, Fadda HM, Basit AW: Gut instincts: Explorations in intestinal physiology and drug delivery. Int J Pharm 364:213-226, 2008.

Patrick KS, Caldwell RW, Ferris RM, Breese GR: Pharmacology of the enantiomers of threo-methylphenidate. J Pharmacol Exp Ther 241:152-158, 1987.

Patrick KS, Gonzalez MA, Straughn AB, Markowitz JS: New methylphenidate formulations for the treatment of attention-deficit/hyperactivity disorder. Expert Opin Drug Deliv 2:121-143, 2005a

Patrick KS, Markowitz JS: Pharmacology of methylphenidate, amphetamine enantiomers and pemoline in attention-deficit hyperactivity disorder. Hum Psychopharmacol 12:527-546, 1997.

Patrick KS, Straughn AB, Minhinnett RR, Yeatts SD, Herrin AE, DeVane CL, Malcolm R, Janis GC, Markowitz JS: Influence of ethanol and gender on methylphenidate pharmacokinetics and pharmacodynamics. Clin Pharmacol Ther 81:346-353, 2007.

Patrick KS, Williard RL, VanWert AL, Dowd JJ, Oatis JE, Jr., Middaugh LD: Synthesis and pharmacology of ethylphenidate enantiomers: the human transesterification metabolite of methylphenidate and ethanol. J Med Chem 48:2876-2881, 2005b.

Ross MK, Crow JA: Human carboxylesterases and their role in xenobiotic and endobiotic metabolism. J Biochem Mol Toxicol 21:187-196, 2007.

Williard RL, Middaugh LD, Zhu HJ, Patrick KS: Methylphenidate and its ethanol transesterification metabolite ethylphenidate: Brain disposition, monoamine transporters and motor activity. Behav Pharmacol 18:39-51, 2007.

Zhu HJ, Patrick KS, Yuan HJ, Wang JS, Donovan JL, DeVane CL, Malcolm R, Johnson JA, Youngblood GL, Sweet DH, Langaee TY, Markowitz JS: Two CES1 gene mutations lead to dysfunctional carboxylesterase 1 activity in man: Clinical significance and molecular basis. Am J Hum Genet 82:1241-1248, 2008.

Address correspondence to:

John S. Markowitz, PharmD

Department of Pharmacotherapy and Translational Research University of Florida College of Pharmacy 1600 SW Archer Road, RM PG-23

Gainesville, FL 32610-0486

E-mail: jmarkowitz@cop.ufl.edu 


\section{This article has been cited by:}

1. Dino Luethi, Philine J. Kaeser, Simon D. Brandt, Stephan Krähenbühl, Marius C. Hoener, Matthias E. Liechti. 2017. Pharmacological profile of methylphenidate-based designer drugs. Neuropharmacology . [Crossref]

2. Hao-Jie Zhu, Kennerly S. Patrick, Arthur B. Straughn, Owen T. Reeves, Hilary Bernstein, Jian Shi, Heather J. Johnson, Joshua M. Knight, Aaron T. Smith, Robert J. Malcolm, John S. Markowitz. 2017. Ethanol Interactions With Dexmethylphenidate and dl-Methylphenidate Spheroidal Oral Drug Absorption Systems in Healthy Volunteers. Journal of Clinical Psychopharmacology 37:4, 419-428. [Crossref]

3. Antonio Molina-Carballo, Ana Checa-Ros, Antonio Muñoz-Hoyos. 2016. Treatments and compositions for attention deficit hyperactivity disorder: a patent review. Expert Opinion on Therapeutic Patents 26:7, 799-814. [Crossref]

4. Kennerly S. Patrick, Timothy R. Corbin, Cristina E. Murphy. 2014. Ethylphenidate as a Selective Dopaminergic Agonist and Methylphenidate-Ethanol Transesterification Biomarker. Journal of Pharmaceutical Sciences 103:12, 3834-3842. [Crossref] 